\title{
IFOSFAMIDE-INDUCED IRREVERSIBLE ENCEPHALOPATHY
}

\author{
Zhang H*, Dong J", Wang $Y^{*}$, Singh S K
}

\section{ABSTRACT}

Ifosfamide associated central nervous system toxicity has been reported in $5 \%$ to $30 \%$ of patients treated with ifosfamide. Death from irreversible encephalopathy has been reported rarely. A 52-year-old lady with carcinoma of cervix was treated with concurrent chemo-Radiatherapy, and residual disease was given with new adjuvant chemotherapy, containing regimens ifosfamide and cisplatin. Two days after the initiation of ifosfamide, the patient exhibited onset of lethal encephalopathy manifested by subacutely progressive encephlopathy then to a vegetative state and eventually to death. Brain CT-scan showed no metastatic tumor and vascular cause, blood routine examination for infection and metabolic factors was also negative; except Urea and Creatinine was increasing. Ifosfamide is known to be capable of causing acute encephalopathy that can be severe but is generally reversible. This lady showed a very atypical progressive, lethal course of ifosfamide toxicity. The possibility of this complication should be considered when kidney function is within normal limits.

\section{Key Words: ifosfamide, encephalopathy.}

\section{CASE REPORT}

A 52-year-old lady was admitted to The Medical Oncology department and diagnosed as carcinoma of the cervix stage IIIB. Three month ago, she has received whole pelvic cobalt60 external beam radiotherapy 46Gy in 23 fractions during 5 weeks with concurrent chemotherapy (cisplatin 30mg per week). Before two month she received Iridium-192 HDR intracavitary brachytherapy 24 Gy in 3 fractions in 3 weeks. After radiotherapy, there was gross cervical residual disease. Then the patient was given chemotherapy. Regimens consisted of 5-FU $1000 \mathrm{mg}$ days 1 to 4 ; cisplatin $100 \mathrm{mg} / \mathrm{m}^{2}$ day 1 . Cycles were repeated every 21 days for 4 courses. The gross residual disease regressed lessly. Planned to change new chemo regimen. Blood test showed: Urea $35 \mathrm{mg} / \mathrm{dl}$, Creatinine $1.6 \mathrm{mg} / \mathrm{dl}$,

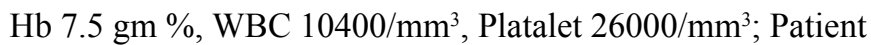
was in good performance status (ECOG, 0-2), had received another chemo-regimen after $1000 \mathrm{ml}$ fresh whole blood. Regimen consist of: cisplatin $50 \mathrm{mg} / \mathrm{m}^{2}$ infusion, day 1 ; Ifosfamide $5000 \mathrm{mg} / \mathrm{m}^{2}$ infusion day 1 for 24 hours with mesna. Two days after the initiation of ifosfamide, the lady exhibited onset of encephalopathy manifested by subacutely progressive disorientation, difficulty with the movements, somnolence and then to coma. The patient's condition did not improve by conservative treatment. Disease progressed to vegetative state and enventually to death seven days after the initiation of ifosfamide. Brain CT-scan showed no metastatic tumor and vascular cause, blood routine examination for infection and metabolic cause was also negative; except Urea $99 \mathrm{mg} / \mathrm{dl}$, Creatinine $2.7 \mathrm{mg} / \mathrm{dl}$.

* B.P. Koirala Memorial Cancer Hospital, Bharatpur, Nepal.

Address for correspondence : Dr. HongZhen Zhang

Medical Oncology Department

B. P. Koirala Memorial Cancer Hospital

P. O. Box: 34, Bharatpur-7, Chitwan, Nepal

Email: hongzhenzhang123@hotmail.com 


\section{DISCUSSION}

Ifosfamide is an alkylating agent used in the treatment of a variety of solid tumor; that acts by DNA crosslinking and chain breakage; Metabolic activation by hepatic microsomal enzymes. Acrolein is highly toxic to urothelial mucosa. The chloroacetaldehyde metabolite may be responsible for much of the neurotoxic effects, particularly in patients with renal dysfunction. ${ }^{1,2}$ Central nervous system (CNS) toxicity of ifosfamide has been reported mostly in the pediatric and adult population. $5 \%$ to $30 \%$ of patient treated with ifosfamide develop an encephathy that can be severe but is generally reversible. ${ }^{3-5}$ The CNS toxicity pattern is characterized by metabolic encephalopathy with confusion, blurred vision, mutism, auditory or visual paranoid hallucinations, seizures, and rarely coma. Ifosfamide in recommended dose cause death is very extremely rare. Data in the medical literature by MEDLINE searches about this subject remains poor. Only a few observations are available. Although Meanwelland Mould and Mould ${ }^{6}$ found two treatment-related deaths in their phase II study of ifosfamide in cervical cancer: one due to leukopeniaassociated infection in a patient with peritonitis and severe central nervous system toxicity and one due to central nervous system toxicity without complicating factors. Since then, Shuper and Stein $^{7}$ reported one case of subacute central nervous system degeneration in a child.

Central nervous system toxicity of ifosfamide is occasional with doses in lower range, more common with large doses, especially when given in 1 day rather than for 5 days and when renal dysfunction is present or when sedatives are given. Renal impairment is occasional to common. Patients must be kept well hydrated with an alkaline $\mathrm{PH}$ to prevent $\mathrm{CNS}$ toxicity and minimize nephrotoxicity. ${ }^{1-2}$ Pelgrims and De Vos et $\mathrm{al}^{3}$ concluded that Methylene blue is an effective treatment for ifosfamide-induced encephalopathy and suggested that it may also be used as a prophylactic agent. Cisplatin and ifosfamide appear to be the best combination therapy in advanced and recurrent cervical carcinoma: they provide an objective response rate of 33\%; combination of cisplatin and 5fluorouracil (5-FU) have been shown to enhance radiation response in several randomized trials. ${ }^{8}$ But in patients with advanced gynecologic malignancies have possible organic or functional disturbance, due to cancer invasion. Occasional advanced cervical cancer patients do not meet the eligibility criteria for chemotherapy because of renal dysfunction due to involvement of ureter. ${ }^{9}$ Bulky pelvic tumor in an area of prior irradiation remains largely refractory to further therapy. ${ }^{10} \mathrm{In}$ our case, patient treated with prior pelvic radiotherapy in addition to cisplatin plus 5-FU whose renal function was within normal limits (Urea $35 \mathrm{mg} / \mathrm{dl}$, Creatinine $1.6 \mathrm{mg} / \mathrm{dl}$ ), was given ifosfamide $5 \mathrm{~g} / \mathrm{m} 2$ iv for 24 hours plus mesna combined with cisplatin $50 \mathrm{mg} / \mathrm{m} 2$ infusion. Two days after the initiation of ifosfamide, the patient developed disorientation, somnolence and coma and eventually to died. Lynch and Ruland ${ }^{11}$ in their article reviewed appropriate ways to monitor for ifosfamide complications such as the kidney, central nervous system (CNS), gastrointestinal tract, and bone marrow and planned correct nursing interventions. They also reported that Ifosfamide metabolite chloroacetaldehyde may be responsible for CNS toxicities. When kidney function and electrolytes are within normal limits and psychotropic medications are not given concomitantly, this rarely occurs. So, This possibility should be considered when kidney function is within normal limits.

\section{REFERENCES}

1. Roland T. Skeel, et al. In Handbook of Cancer Chemotherapy. Fifth Edition.1999;Aatineoplastic drugs and biologic response modifiers, Ifosfamide.111-12.

2. Dennis A. Casciato, et al. In Manual of Clinical Oncology. Fourth Edition.1999; Chemotherapeutic Agents, Ifosfamide. 57-58.

3. Pelgrims J, De Vos F, et al. Methylene blue in the treatment and prevention of ifosfamide-inducedencephalopathy: report of 12 cases and a review of the literature. Br J Cancer 2000; 82(2): 291-94.

4. Anderson NR, Tandon DS. Ifosfamide extrapyramidal neurotoxicity. Cancer 1991; 68(1): 72-75.

5. Bhardwaj A, Badesha PS. Ifosfamide-induced nonconvulsive status epilepticus. Ann Pharmacother, 1995; 29(12): 123739.

6. Meanwell CA, Mould JJ, et al. Phase II study of ifosfamide in cervical cancer. Cancer Treat Rep, 1986; 70(6): 727-30.

7. Shuper A, Stein J, et al. Subacute central nervous system degeneration in a child: an unusual manifestation of ifosfamide intoxication. J Child Neurol, 2000; 15(7): 481-83.

8. Nguyen HN, Nordqvist SR. Chemotherapy of advanced and recurrent cervical carcinoma. Semin Surg Oncol, 1999; 16(3): 247-50.

9. Shimizu Y. Consecutive low-dose cisplatin-based chemotherapy for gynecologic malignancies. Gan To Kagaku Ryoho, 1997; 24(4): 431-38.

10. Alberts DS, Garcia DJ. Salvage chemotherapy in recurrent or refractory squamous cell cancer of the uterine cervix. Semin Oncol, 1994; 21(4 Suppl 7): 37-46.

11. Lynch MP, Ruland T. Ifosfamide. Patient care management. Cancer Nurs, 1993; 16(5): 362-65. 